Thorax (1948), 3, 71.

\title{
THE TREATMENT OF PULMONARY HYDATID DISEASE
}

\author{
BY \\ M. P. SUSMAN \\ From the Sydney Hospital and the Royal North Shore Hospital, Sydney, Australia
}

There is at present no medical treatment for hydatid cysts, wherever they may be situated. Some, especially in the liver, die and become harmless calcified masses. Some in the lung may be completely coughed up without the occurrence of complications, and perfect cure may follow. Dew and Devé, among others, believe that more people than we know of cough up their cysts and never seek medical advice. The following case records are to the point.

Case 1.-A boy aged 9 years had three attacks of haemoptysis within three months. A radiograph showed an irregular opacity in the upper part of the right lung, and the Casoni and complement fixation tests were positive. He had several more haemorrhages during the following year, but was not brought to hospital until he had coughed up hydatid membrane and fluid and more blood. A radiograph taken soon after the episode was clear, and fifteen months later he was still well, with a normal radiograph.

Case 2.-Four years ago a man aged 19 had the first stage of an operation for a cyst in the right upper lobe. While awaiting the second stage he coughed up his cyst without incident and has been well ever since.

But intrabronchial rupture of a hydatid cyst involves many risks, such as anaphylaxis, asphyxia, haemorrhage, and suppuration. There is also the danger of rupture into the pleural cavity or heart or into a great vessel. Because of these possibilities I think that all hilar cysts, unless small, as well as all peripheral cysts should be treated promptly by operation. The small hilar cysts, if not coughed up in the meantime, should be treated surgically when they become about two inches in diameter; by this time they are present on the surface of the lung and many are already close to the chest wall.

\section{Operative Treatment: Personal Experience}

The operative treatment of hydatid cysts of the lung is still not standardized in spite of the collective experience of many surgeons over many years. The following observations are based on twentyseven personal cases and a review of forty-five more from the records of Sydney Hospital and Royal North Shore Hospital, Sydney, Australia.
The aims of conservative surgery are to remove the cyst completely so that only the adventitia remains, to avoid implantation of hooklets and scolices, and to prevent complications such as infection of the pleural cavity and lung, tension pneumothorax, atelectasis, and delayed re-expansion of the lung.

The following experience of post-operative infection of the pleura was especially disturbing.

Case 3.-A man aged 19 was recommended for treatment because of an opacity in the left lung in a routine radiograph (Plate XIVa). A one-stage operation was done through a free pleural space, the adventitia was drained and sewn to the chest wall, and the pleura was closed without drainage. The lung re-expanded at once, but from the time of operation the patient was ill, with signs of infection. A severe empyema with Bacillus proteus developed, and clubbing of the fingers was soon apparent. For many weeks he was dangerously ill, but he eventually recovered completely after months of drainage.

Adhesions may be lacking even in the presence of a suppurating cyst.

Case 4.-A girl aged 16 suddenly and without warning coughed up some watery fluid ; this was followed by fever and the expectoration of purulent sputum. I saw her a month later, when the outstanding signs were dullness and absent breath sounds at the right base. She brought a radiograph with her showing a round area in the right lower lobe, with an opacity at its bottom (Plate XIII $a$ ). A new radiograph showed that the lesion was much bigger and had a fluid level; a slight irregularity on the surface of the fluid was probably due to the opacity noted in the first film (Plate XIIIb and c). At operation I found a free pleural space; the cyst was packed off and brought well into the wound for aspiration and opening. Pus and crumpled membranes were removed without soiling the pleura, and the cyst space was drained after the adventitia had been sutured to the chest wall. The pleural cavity was closed without drainage. Plate XIIId is a radiograph taken two weeks after operation. The patient was discharged several weeks later with the wound healed and the radiograph showing only a vague opacity.

It is when there are no adhesions, as in these cases, that difficulties and dangers are most apt to occur, and this at once suggests that the obvious procedure is to induce adhesions before attacking 
the cyst. But, as most thoracic surgeons have found, it may be impossible to produce satisfactory adhesions whatever method is tried, so that one often ends by doing a delayed one-stage operation instead of the contemplated two-stage operation.

Case 5.-A woman aged 25 suddenly coughed up some watery fluid and later some blood. She became breathless, and her doctor found that she had "acute oedema of the lungs due apparently to rupture of a hydatid cyst." When I saw her several weeks later she had recovered from this attack, and a radiograph showed a round opacity in the right lower lobe (Plate XIVb). A two-stage operation was done, with an interval of two weeks between stages. The lung was still not adherent at the second stage but the operation was completed. The fluid in the cyst was turbid and both the cyst space and the pleural cavity were drained; the lung re-expanded rapidly and the cyst space was soon obliterated. Four years later I had a report that she had remained well.

When the cyst is adherent to the chest wall (and it may be even if the cyst is not infected) there is no problem, provided that the surgeon has accurately localized the site of adhesion; it is a simple matter to open the cyst, remove the contents, and close the adventitia or put in a tube, depending on the absence or presence of infection. If the free pleural space is opened close to the adherent cyst, either by chance or on purpose, one notes the exact site of adhesion with a finger in the pleural cavity, then closes the pleura securely and opens the adherent cyst with certainty at the right spot.

Case 6.-A man aged 22 joined the Army with a clear chest radiograph. Three years later he had a fever with bloody sputum and pain in the left axilla. There was a hydatid cyst about 3 in. in diameter in the left upper lobe, and at operation the pleura was accidentally opened close to the site of the adhesion. The pleura was at once closed and the cyst emptied and drained through the adherent zone. Convalescence was uneventful.

I now do a one-stage operation whether the cyst is adherent or not, and this is in agreement with the views of many surgeons, past and present. Some of the older school of Australian surgeons dropped the lung back after emptying an unadherent cyst and closed the chest without drainage. It is hard to come by exact figures of their results, but to drain neither the cyst space nor the pleural cavity would, from my experience, increase the risk of complications. However, two Spanish surgeons (G. Mauresa, of Barcelona, and L. Escudero Bueno, of Madrid) claim good results with this method of treatment (personal communications, 1947). They have had few complications and no serious residual symptoms, although some patients have shown radiological evidence of a residual cystic space. Neither of them has performed lobectomy for hydatid disease either as a primary or secondary operation.

Dew (1928) states the case for draining the marsupialized cyst and closing the pleura. Most of the patients I have so treated have done well, and Officer-Brown (1942) in a series of twenty cases was well satisfied with this line of treatment ; he had no deaths, few serious complications, and good end-results. Dew adds that it is sometimes necessary to drain the pleural cavity also if it has been contaminated by hydatid fluid. My present aim, like Barrett's, is to close the cyst space and to leave a tube in the pleural cavity for closed drainage ; but I disagree with Barrett's (1947) statement that it is unsafe to fix the adventitia and lung to the chest wall because of the risk of their tearing away. If several inches of one or, if necessary, of two ribs are removed for the thoracotomy there is more soft tissue available and suturing is easy and accurate.

For a simple unadherent cyst I advise removal of the cyst contents ; closure of the adventitia with interrupted sutures, the ends of which are passed through the intercostal muscles and tied again so as to bring the closed adventitia up to the parietal pleura ; the reinforcing of these with several more stitches so that the pleural cavity is completely closed ; and sealed drainage of the pleural cavity through a separate wound. If the cyst is so situated that the closed adventitia cannot be sutured to the chest wall, I allow the lung to drop back, and drain the pleura. The reason for fixing the closed adventitia to the chest wall is that if any complication occurs in the cyst space, such as haematoma or infection, it is immediately accessible for reopening. The objects of draining the pleural cavity are to obtain prompt re-expansion of the lung and to anticipate possible infection or tension pneumothorax. If an unadherent cyst is already infected it should be drained and marsupialized; I have sometimes, in these circumstances, closed the pleural cavity without drainage, as in Case 4 above, but closed drainage of the pleural cavity is, I think, the best procedure here too.

\section{Pre-operative Aids}

There are several points about pre-operative aids that I wish to discuss.

1. Bronchograms in my experience show no abnormality with an uncomplicated cyst except the filling defect of the cyst's opacity and some crowding of the small bronchi. After rupture of the cyst and infection there may be some bronchiectasis, but it is rarely extreme enough to justify lobectomy unless causing frank symptoms. Gross 


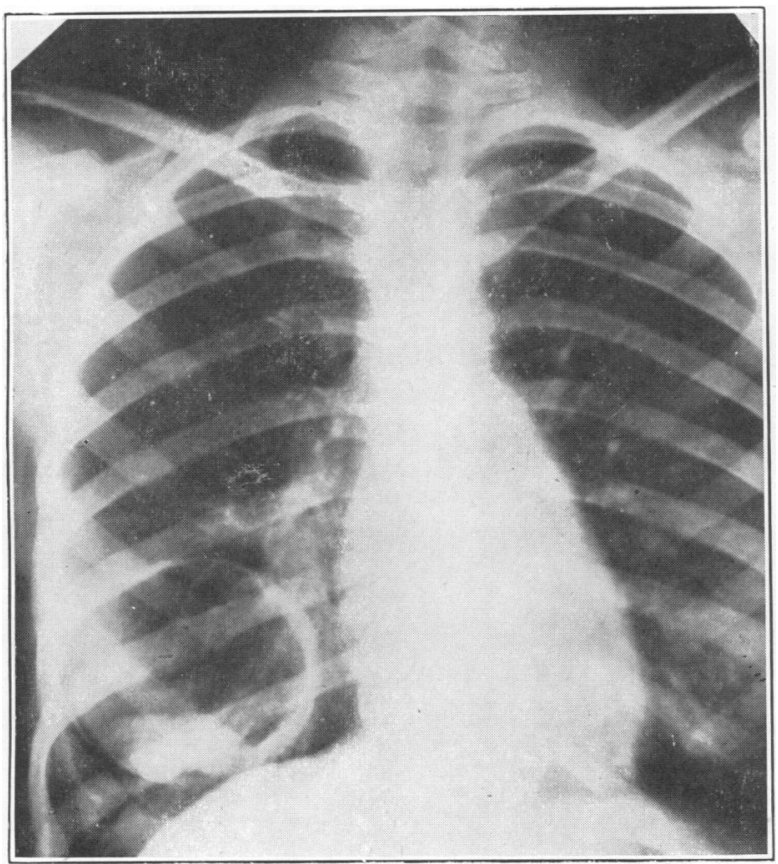

(a)

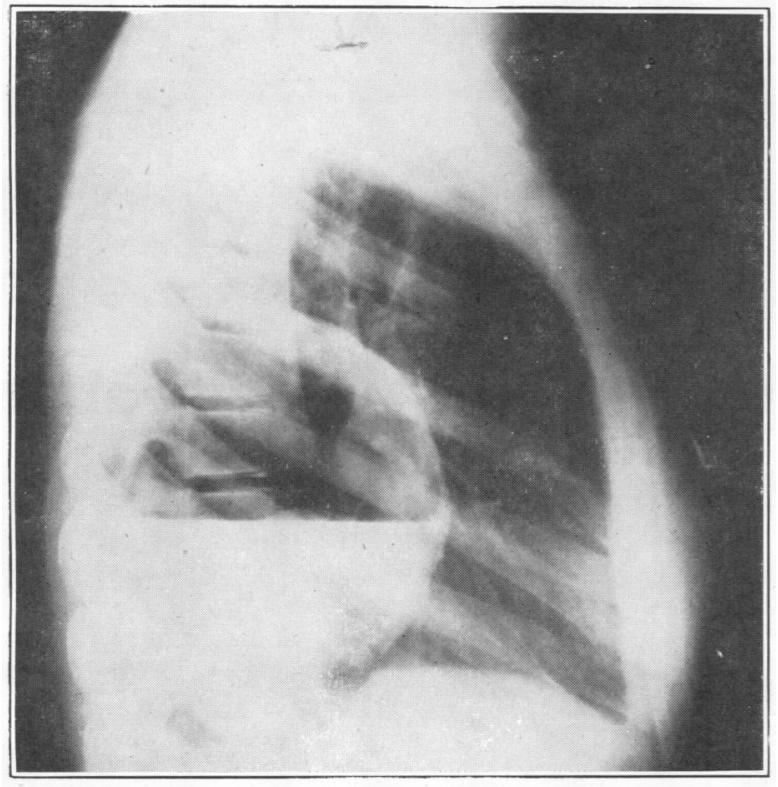

(c)

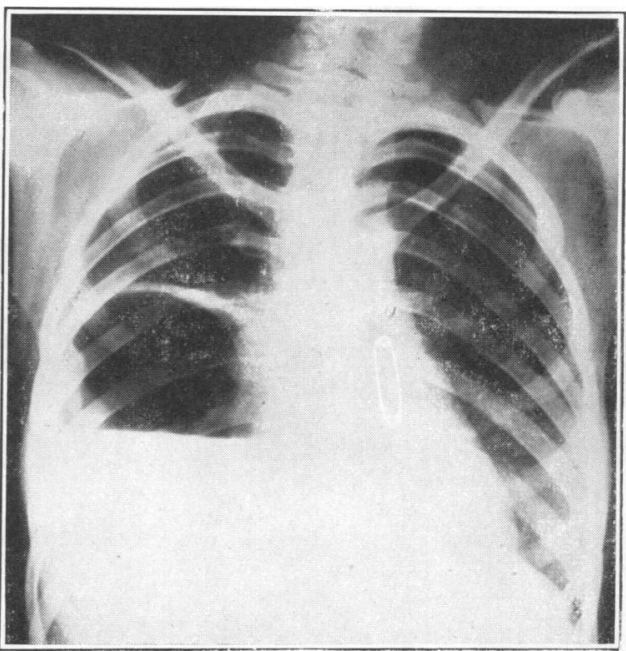

(b)

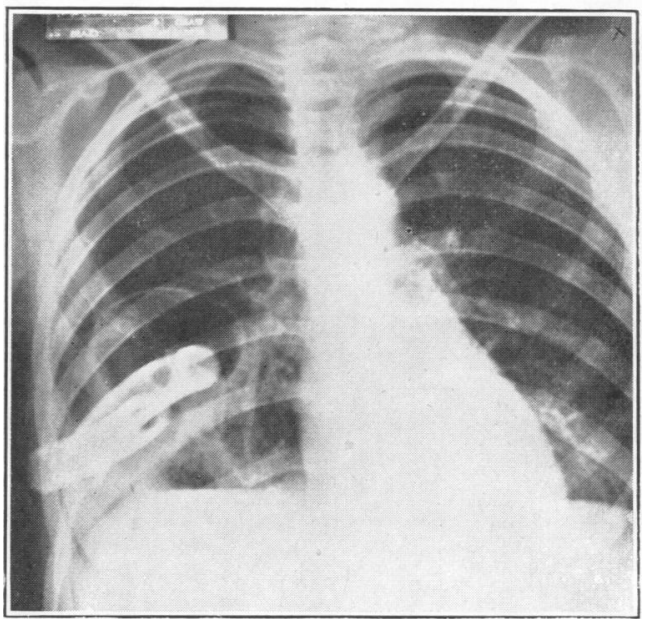

(d)

Plate XIII.-Case 4. (a) Cyst after fluid has been coughed up. The collapsed ectocyst is at the bottom of the space. (b) Distended cyst with fluid level; the slight irregularity on the surface is the top of the collapsed ectocyst. (c) Lateral view, showing the posterior position of the cyst. (d) Radiograph taken two weeks after operation. The two drainage tubes are both in the large cyst space. 


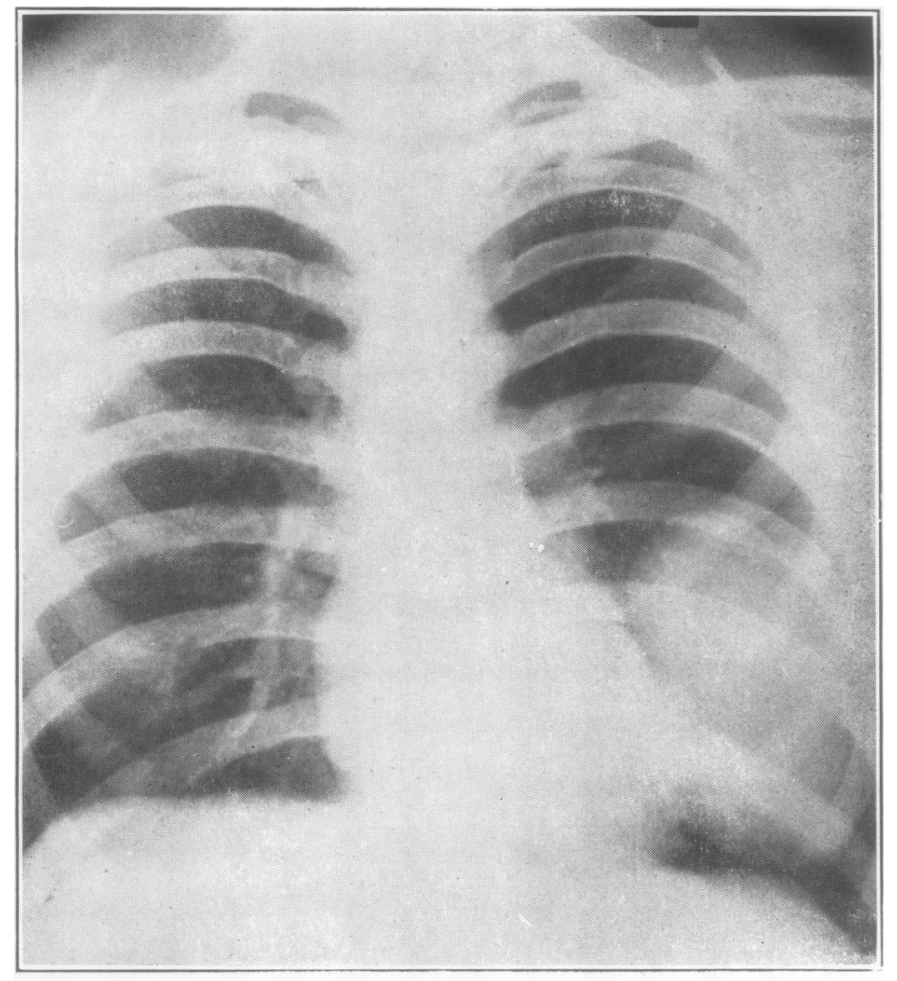

Plate XIV._- (a) Case 3: Uninfected hydatid cyst of the left lower lobe. (b) Case 5: Radiograph taken several weeks after some of the cyst contents had been coughed up. (c) Case 7 : Lateral view of bronchogram. The enlarged middle lobe contains an air-filled cavity. No oil has entered the middle lobe.

(a)

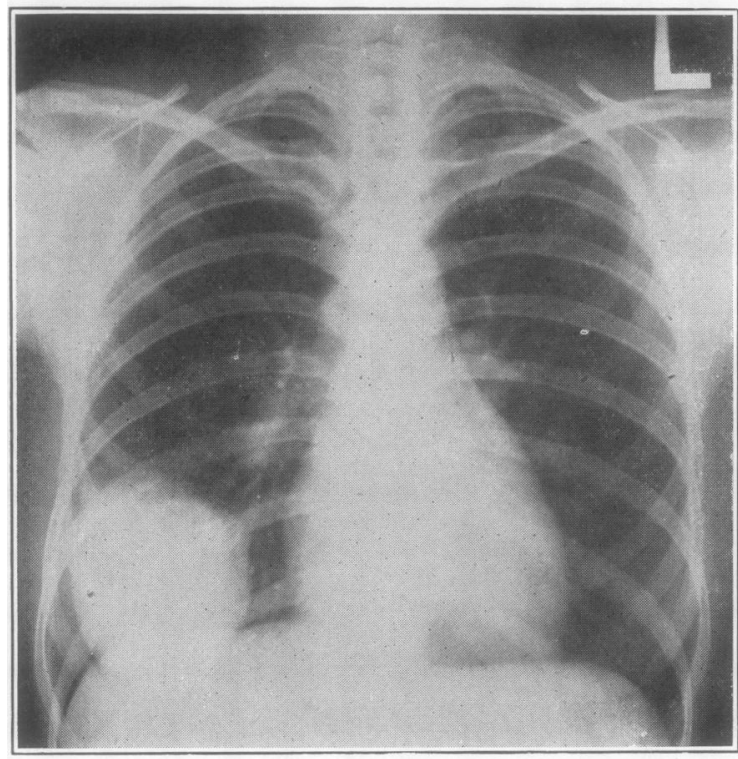

(b)

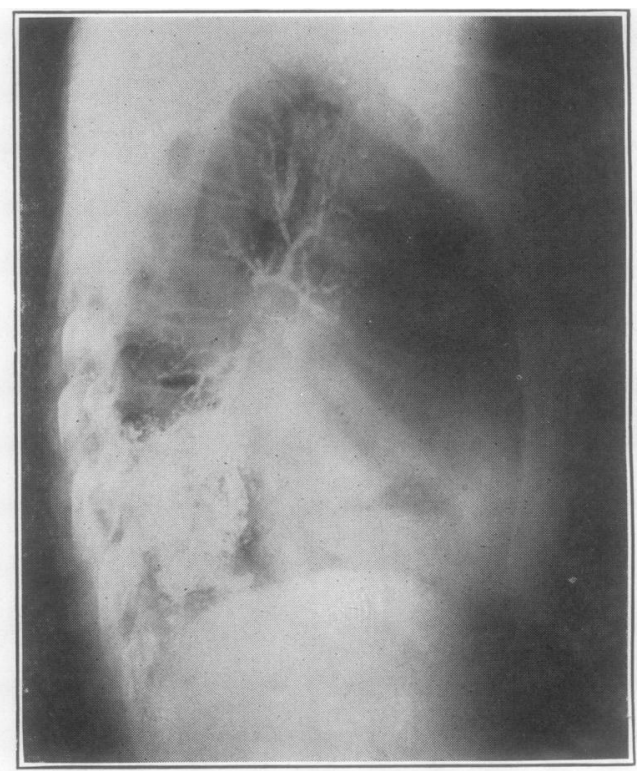

(c) 


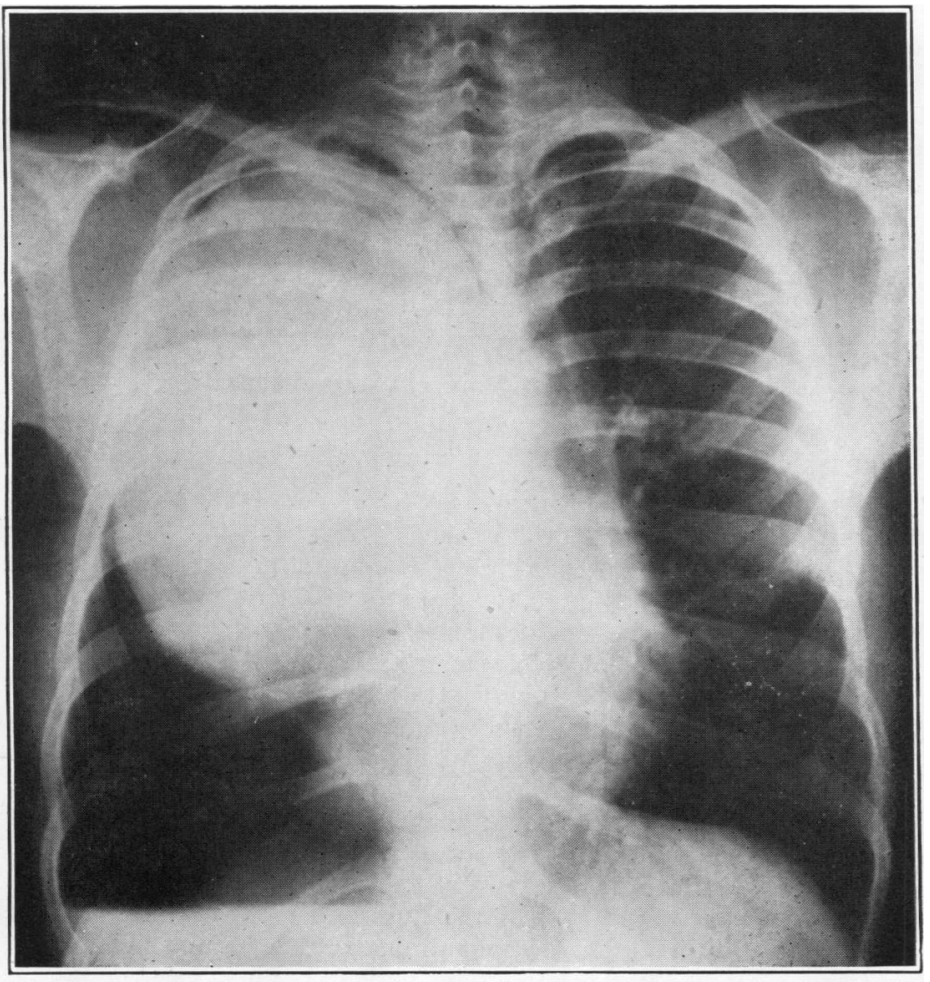

Plate XV.-(a) Case 8 : Giant hydatid cyst of the left lung (the film has been reversed). (b) Case 9: Bilateral hydatid cyst. (c) Case 13: Hydatid cyst of left lower lobe.

(a)

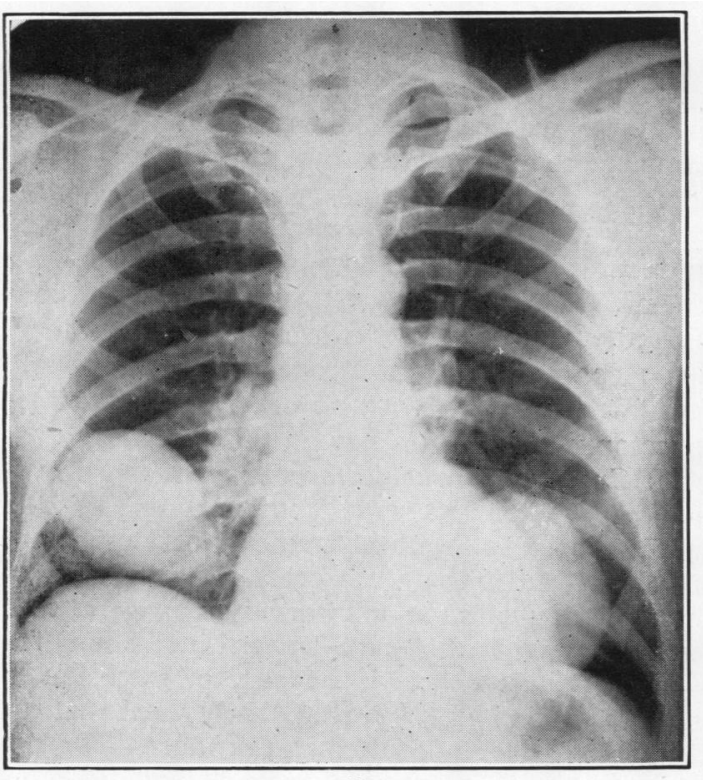

(b)

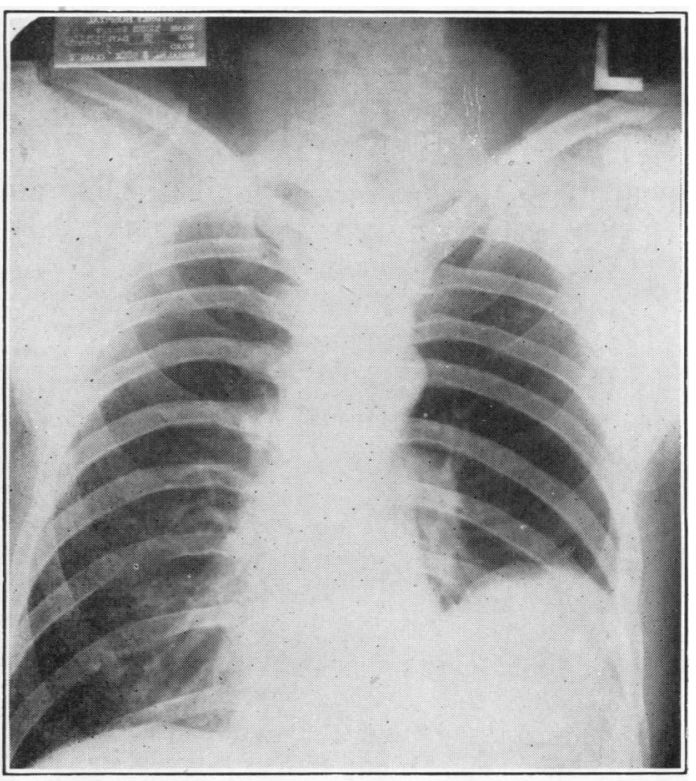

(c) 
residual damage usually means that there has been incomplete emptying of the cyst, whether by coughing or by operation (see Cases 7 and 11).

2. A preliminary pneumothorax is necessary if one decides to promote adhesion, before attacking the cyst, by injecting silver nitrate or other irritant into the pleural cavity. It may also be used to aid localization if the cyst is already adherent. But nothing is gained by it if a one-stage operation is intended and the cyst is not adherent ; it may even be dangerous, as I have known an experienced house surgeon to puncture the cyst while attempting induction in an apparently safe spot.

3. Bronchoscopy may complete the diagnosis in a doubtful case after unrecognized rupture of a cyst. It may also have some therapeutic value.

Case 7.-A woman aged 57 was admitted to hospital with "pneumonia and empyema"; according to the hospital notes one aspiration of $16 \mathrm{oz}$. of pus was the only treatment she had for the "empyema." Two months later she had an attack of "pleurisy and haemoptysis," from which she made an incomplete recovery. Six months later she returned because of persistent pain in the right side of the chest and a cough productive of about $4 \mathrm{oz}$. of purulent blood-stained sputum daily; no hydatid elements were found in it, and the Casoni test was negative. One radiograph showed a cavity, with a fluid level, and in a bronchogram it was noted that no oil entered the middle lobe and that this lobe was enlarged and contained the cavity (Plate XIV c). At bronchoscopy I saw some pus in the middle lobe bronchus and a foreign body protruding from its orifice ; microscopically this proved to be hydatid membrane. She improved after several bronchoscopic aspirations, and three years later she was well except for cough and a little sputum.

\section{LOBECTOMY FOR HYDATID CYST}

I think that the indications for lobectomy are few. Before giving my own, I wish to discuss those cited by Barrett (1947), namely:

(a) Large cyst oscupying most of the lobe.

(b) Cyst with tough adventitia.

(c) Cyst complicated by bronchiectasis or pulmonary suppuration.

(d) Dead cyst.

(e) Empty sac.

(a) Large cyst occupying most of the lobe.I doubt whether the size of the cyst is of itself an indication for lobectomy. The dead space left after emptying the cyst is filled by expanding lung just as after a lobectomy ; even with a giant cyst some lung tissue in the involved lobe is still healthy though compressed, and this will function again once the mechanical hindrance of the cyst has been removed. To save any healthy lung tissue, except when dealing with cancer, is now the surgical aim, and permanent changes around a simple cyst are rare. Even when the cyst has suppurated the surrounding lung tissue is not seriously altered unless the infection has been severe enough to cause sloughing of the adventitia ; then there will be the usual evidence of gross pulmonary suppuration for which excision may be the only cure (see paragraph $c$ below). There is, however, the risk of anaphylaxis to be considered. Probably the larger the cyst the greater the risk, but does anyone know whether the risk of fatal anaphylaxis after direct operation on a cyst is greater than the risk of lobectomy with the cyst intact? Certainly the following case could be used in support of lobectomy for a giant cyst.

Case 8.-A boy aged 17 complained of pain in the left side of his chest and mild dyspnoea. These symptoms soon abated, and he did not come under my care until many months later, when there were clinical and radiological signs of a large mass in the left hemithorax with moderate displacement of the mediastinum to the right (Plate XVa). The Casoni test was negative, but the precipitin and complement fixation tests were positive. A one-stage operation was done without incident; the cyst was not adherent but it bulged into the wound as soon as the pleura was opened. The adventitia was sewn to the chest wall after removal of the fluid and membrane, the pleura was closed without drainage, and a tube was left in the cyst cavity. The patient became very ill during the night and went from bad to worse, with high, irregular fever, rapid pulse, and coarse rales in the right lung; the left lung failed to re-expand. Jaundice appeared shortly before he died on the ninth day after operation. There was no autopsy.

(b) Cyst with tough adventitia.-Even when the adventitia is tough it is rare, in my experience, for the cyst space to remain unobliterated after complete removal of the cyst contents and after adequate drainage if it has been infected. It may te necessary to remove segments of several ribs to secure proper drainage of an old cyst with chronic infection. Lobectomy should be considered only when adequate drainage has failed, and even then a local thoracoplasty would probably be sufficient. I have had no occasion to do either lobectomy or thoracoplasty on this account.

(c) Cyst with bronchiectasis or pulmonary suppuration.-Bronchiectasis complicating hydatid cyst is usually so slight that radical operation is not required; but if there are gross symptoms lobectomy, of course, is the only remedy. I do not ascept suppuration of the cyst as an indication for lobectomy unless there is evidence of extension to the surrounding lung. Barrett (1947) states that the cyst " may become infected and produce a chronic lung abscess containing sphacelated hydatid rem- 
nants surrounded by spreading pulmonary suppuration." In my experience, it is rare to have gross, irrecoverable infection around a suppurating cyst, and I think it is wrong to regard an infected cyst as pathologically similar to an ordinary chronic lung abscess. The adventitia around the cyst makes all the difference, and it is only when this fails to act as a barrier that we should treat the condition as one of true chronic lung abscess ; then, of course, resection is often the only cure. Most of the patients with suppurating cysts do well with simple drainage-for examples, Case 5 above and the two following cases.

Case 9.-A man aged 60 came to hospital with pulmonary and pleural infection, having 3 to $4 \mathrm{oz}$. of purulent sputum daily. A radiograph taken three years before this had shown hydatid cysts in both lungs, but he had had no treatment for them $($ Plate XVb). Now he had a pleural effusion with a fluid level obscuring the left lung field, and a circular opacity in the right lung. The first operation was for the drainage of a left empyema through the tenth rib bed. Two weeks later a segment of the eighth rib was removed for drainage of a suppurating hydatid cyst in the left lower lobe. He did well after these operations and returned eight months later for treatment of the cyst of the right lung. At operation the right lower lobe fell away when the pleura was opened, and a partly adherent mass was felt in the middle lobe close by. The pleura was closed and normal lung around the mass was stitched to the chest wall. The opening of the cyst was deferred for three weeks, when collapsed endocyst and pus were found. The cyst space was drained and healing occurred in four weeks.

Case 10.-A woman aged 31 had a suppurating cyst about 3 in. in diameter in the right lower lobe, with offensive sputum, clubbed fingers, and mild constitutional upset. A bronchogram showed no bronchiectasis and no oil entered the opacity. At operation the cyst was found firmly adherent and offensive pus and old hydatid membrane were removed. The cavity was drained and complete healing took four months. Her general health improved rapidly after the operation, and the final radiograph was normal except for a streaky opacity.

Barrett's reasons for advising lobectomy for an infected cyst are:

1. That a permanent sinus or lattice lung may follow drainage.

2. That "the lesion found in the resected part after lobectomy has always been so extensive as to be incurable by other means."

3 . That the mortality rate after drainage is 20 or 30 per cent according to Dew.

In answer to these points :

1. I have found that a permanent sinus is rare if there has been adequate drainage; in the following case, for instance, a sinus remained, after the first operation. At a later exploration part of the endocyst was found and prompt healing followed its removal.

Case 11.-A boy aged 12 had an operation for supposed lung abscess or empyema three months before I saw him. The tube had been left in for four weeks and the wound had healed temporarily, but when he came to me he had a discharging sinus. Radiographs showed an irregular opacity at the right base, and iodized oil injected into the sinus showed a cavity with a bronchial fistula. After excising the sinus and a segment of rib I found a cavity containing collapsed hydatid membrane and some debris. Healing occurred after seven weeks of drainage.

I have never seen a lattice lung secondary to hydatid disease, nor could I find an example of it in the records at Sydney Hospital and Royal North Shore Hospital between the years 1922 and 1946.

2. In my experience severe bronchiectasis and pulmonary suppuration rarely complicate a hydatid cyst, and I think that lobectomy is indicated only if there are appreciable symptoms, after drainage of a suppurating cyst, or emptying of a simple cyst.

3. The latest edition of Dew's book is dated 1928 , and the mortality nowadays after drainage of an infected cyst is nearer zero than 20 per cent. Neither Officer-Brown nor I had a death from sepsis, and there were no post-operative deaths from sepsis in the hospital series of cases that have been reviewed.

(d) Dead cyst.-Many people harbour dead cysts without danger or inconvenience, and many cysts have been found unexpectedly at autopsies. Radical operation for dead cyst of itself is not justifiable. If there are symptoms, removal of the contents and adequate drainage will usually suffice; should this conservative treatment fail, lobectomy may be necessary.

(e) Empty sac.-An empty sac without symptoms does not call for operation. If there are any symptoms, such as infection or haemorrhage, each case should be assessed individually.

Case 12.-A woman aged 32 coughed up a hydatid cyst of the left upper lobe. She remained well for a year and then began to suffer from recurrent haemoptyses. As the haemorrhages were becoming more severe I removed the involved lobe. It harboured an irregular cavity partly filled with blood clot and debris. She is now well and symptomless.

My indications for lobectomy in hydatid disease are:

1. Serious haemorrhage at operation undertaken ptimarily for removal of the cyst contents. 
I have never had to perform lobectomy because of this, but I had to consider it at one operation.

Case 13.-A man aged 50 failed to recover completely from a left basal pneumonia. A radiograph showed a circular opacity at the left base (Plate XVc) and bronchograms showed that no oil entered the opacity or the posterior basal bronchus. Nothing abnormal was found at bronchoscopy. At operation a tense adherent cyst was found without any infection. Free bleeding occurred from the cavity, but no blood entered the bronchial tree. A transfusion was started and I was considering lobectomy when the haemorrhage began to diminish, so I ended the operation by packing the cavity. The packing was removed two days later and there was no further trouble.

Alarming haemorrhage may follow the opening of any hydatid cyst, and the surgeon should always be ready for it.

2. Residual bronchiectasis, after the cyst has been emptied by an earlier operation or by coughing, if symptoms are severe.

3. Empty sac complicated by haemorrhage or infection.

4. Uncertain diagnosis-for example, if there is a strong suspicion of carcinoma.

5. A possible indication is giant cyst, because of the risk of serious anaphylaxis if the cyst is attacked directly.

\section{SUMMARY}

1. The treatment of hydatid cysts of the lungs is discussed on the basis of a review of 27 personal cases and of 45 more from the records of the Sydney Hospital and the Royal North Shore Hospital, Sydney. Some require no treatment, but for the majority operation is necessary.

2. As a rule a conservative type of operation suffices, and the procedure adopted may be summarized as follows:

A. When the lung is adherent to the chest wall:

$a$. If the cyst is not infected, the contents are removed, and the adventitia is closed.

$b$. If the cyst is infected, the contents are removed, and the cyst space is drained.

Should the pleural cavity be entered during the operation, it must be securely closed before opening the adherent cyst.

B. When the lung is not adherent:

a. If the cyst is not infected, the pleural cavity is freely opened and protected, the contents of the cyst are removed, the adventitia is closed and sutured to the chest wall, and the pleural cavity is drained, with a water seal, through a stab wound. $b$. If the cyst is infected, the pleural cavity is opened and protected, the contents of the cyst are removed, the cyst space is drained and marsupialized, and the pleural cavity is drained through a stab wound.

3. Lobectomy may be necessary for the following conditions :

1. Serious haemorrhage from the cyst space during a conservative operation.

2. Residual bronchiectasis, if symptoms warrant it.

3. Empty sac, if haemorrhage or infection warrant it.

4. When the diagnosis is uncertain.

5. Giant cyst.

\section{Appendix: Review of Material}

In my series of twenty-seven cases twenty-three were treated by operation.

One was treated by lobectomy because of haemorrhage following the coughing up of the cyst (Case 12).

One had the first stage of thoracotomy and coughed up the cyst while awaiting the second stage (Case 2).

Twenty-one were treated by removal of the cyst contents, followed by drainage of the cyst space or of the pleural cavity or of both.

Four had no operation. These four patients coughed up their cysts. One should have had operative treatment but refused. One recovered after removal of hydatid membrane and pus by bronchoscope (Case 7); one had a mild bronchiectasis not requiring lobectomy ; and one was apparently cured (Case 1).

Of the twenty-seven, one patient died (Case 8); death was probably due to anaphylaxis after removal of the cyst contents and drainage of the adventitial space.

In the series of thirty-five cases from the Sydney Hospital records there were four deaths. Two of these occurred without operation apparently from sepsis and cardiac failure (in 1928 , so that no chemotherapy was available). The other two deaths were after operation; one from immediate surgical emphysema and shock (no autopsy), and one from bronchopneumonia one month after emptying of an uninfected cyst (proved at autopsy).

In the series of ten cases from the Royal North Shore Hospital all were treated by removal of the cyst contents and drainage of the adventitial space. There was one death, probably due to tension pneumothorax.

I have to thank Drs. W. Freeborn, N. Rose, and P. Greenwell for help with the hospital records.

\section{REFERENCES}

Barrett, N. R. (1947). Thorax, 2, 21 , Dew, H. (1928). "Hydatid Disease." Sydney: The Australian Medical Publishing Company.

Officer-Brown, C. J. (1942). Royal Melbourne Hospital Reports, Vol. 13. 